\title{
ATOMISTIC SIMULATION OF THE UNIAXIAL TENSION OF BLACK PHOSPHORENE NANOTUBES
}

\author{
Van-Trang Nguyen ${ }^{1,2}$, Danh-Truong Nguyen ${ }^{1}$, Minh-Quy Le ${ }^{1, *}$ \\ ${ }^{1}$ Hanoi University of Science and Technology, Vietnam \\ ${ }^{2}$ Thai Nguyen University of Technology, Thai Nguyen City, Viet Nam \\ *E-mail: quy.leminh@hust.edu.vn \\ Received September 23, 2017
}

\begin{abstract}
In the present work, the uniaxial tensile tests of 3 pairs black phosphorene nanotubes are simulated by molecular dynamics finite element method with Stillinger-Weber potential. Each pair contains an armchair black phosphorene nanotube and a zigzag one, which have approximately equal diameters. Three armchair black phosphorene nanotubes, namely $(0,15),(0,19)$ and $(0,21)$; and three zigzag black phosphorene nanotubes, namely $(20,0),(26,0)$ and $(28,0)$, are considered. We found that the Young's modulus of these tubes increases when their diameter increases. Their fracture stress and fracture strain are also investigated.
\end{abstract}

Keywords: 2D material, atomistic simulation, phosphorene nanotube, mechanical properties.

\section{INTRODUCTION}

Two-dimensional (2D) black phosphorous, namely, back phosphorene or $\alpha$-phosphorene $(\alpha-\mathrm{P})$ has been recently synthesized [1-3]. $\alpha$-P is a semiconductor with a large direct band gap, see e.g. [4], and has potential applications in different fields such as nanoelectronics, optoelectronics [2,4,5], gas sensors [6], and Li-ion batteries [7,8]. Therefore, the mechanical properties of $\alpha$-P nanostructures need to be studied. A $\alpha$-P nanotube can be constructed by rolling up a $\alpha$-P monolayer along the armchair and zigzag direction (see Fig. 1).

Chen et al. [9] performed molecular dynamics (MD) simulations with compass force field and showed that Young's modulus of both armchair and zigzag $\alpha$-P nanotubes could increase with the tube length and diameter. Density functional theory (DFT)-tight binding calculations by Sorkin et al. [10] showed that fracture stress and Young's modulus of armchair $\alpha$-P nanotubes are larger than those of zigzag $\alpha$-P ones. Ansari et al. [11] used beam elements with harmonic potential to model bonds and revealed that phosphorene nanotubes with larger diameter have larger Young's modulus. However, limitations of

(C) 2018 Vietnam Academy of Science and Technology 


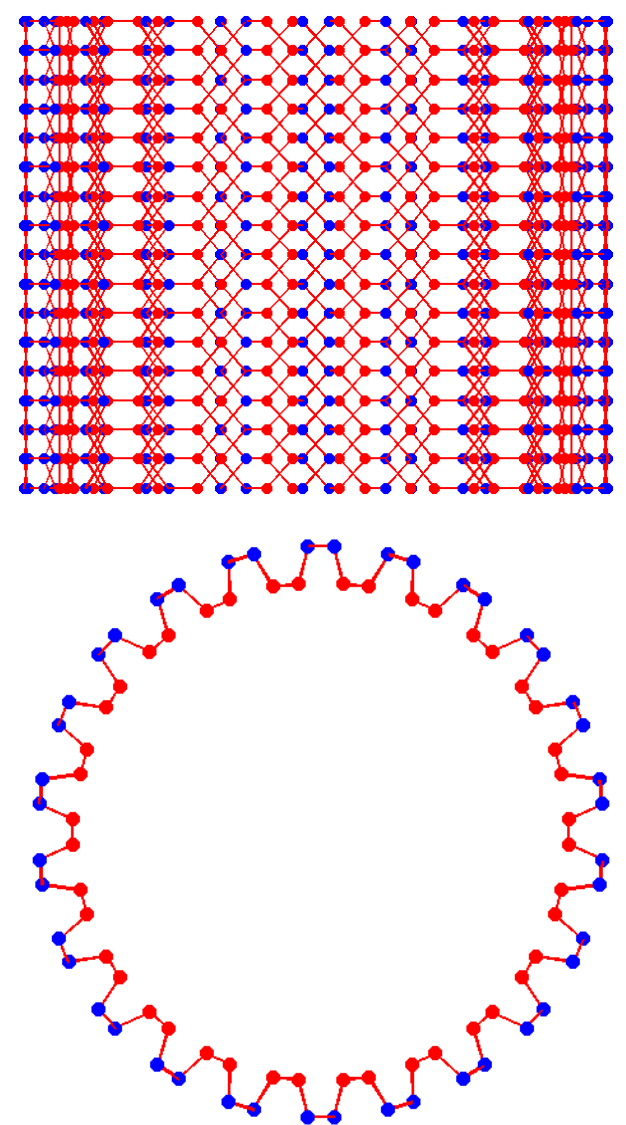

(a)
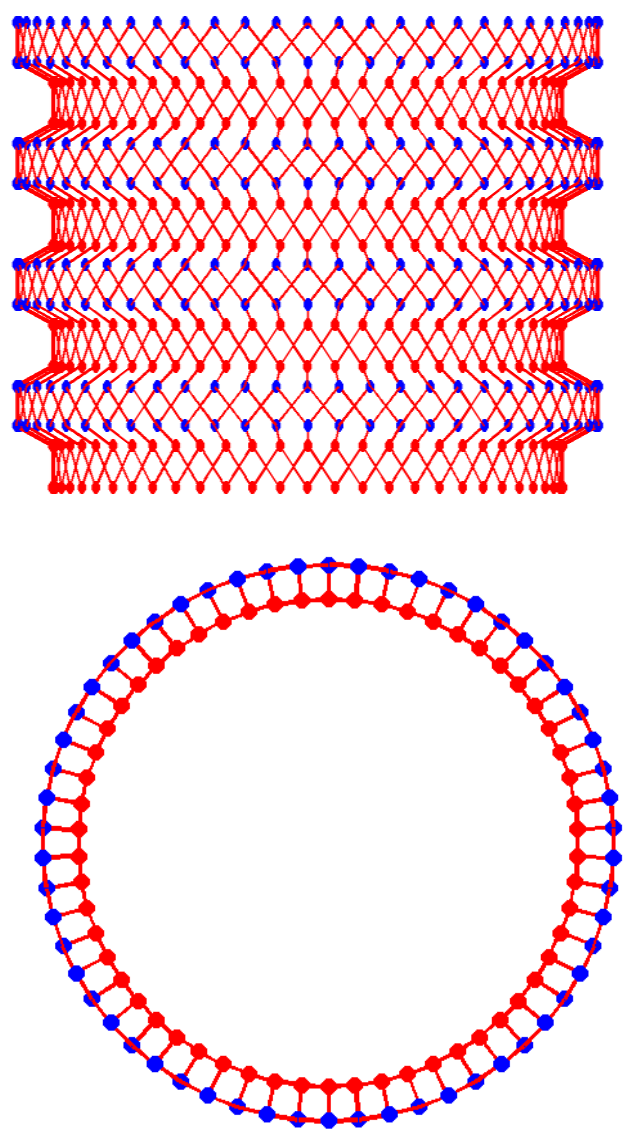

(b)

Fig. 1. Schematic illustration of: a) armchair $\alpha$-P nanotube; b) zigzag $\alpha$-P nanotube

beam models are clear since bond between 2 neighbor atoms is always strait and never bent. Further limitations of the beam models have been discussed in [12]. It should be noted that fracture of $\alpha$-P nanotubes cannot be studied by the compass force field, which was used in [9], and by the harmonic force field, which was used in [11], because the bond energy computed from the compass or harmonic force field becomes infinite when bond extension tends to the infinite.

Wei et al. [13] reported that a phosphorene sheet can withstand tensile strain up to $27 \%$ and $30 \%$ in the zigzag and armchair directions, respectively. The temperaturedependent stress-strain relations of $\alpha$-P sheet under uniaxial tension were previously investigated [14]. It is shown that the Young's moduli, fracture strength and strain slowly decrease with increasing in temperature $[14,15]$. In addition, the mechanical properties of $\alpha$-P sheet have been investigated by DFT calculations [16,17] and MD simulations [18].

In the present work, the uniaxial tensile tests of 3 pairs of $\alpha$-P nanotubes are simulated by molecular dynamics finite element method (MDFEM) with Stillinger-Weber 
potential. Each pair contains an armchair $\alpha$-P nanotube and a zigzag one, which have approximately equaled diameters. The present method is of course more reliable than finite element analysis with beam elements [11] as above-mentioned. Our method allows predicting the mechanical properties up to fracture that could not be considered in $[9,11]$.

\section{FRAMEWORK FOR ANALYSIS}

In this work, Stillinger-Weber potential is used to model the interatomic interactions [19]. The potential energy $E$ of the atomic structure is the sum of the bond stretching energy $E_{r}$ and bond angle bending energy $E_{\theta}$

$$
\begin{gathered}
E=E_{r}+E_{\theta}, \\
E_{r}=\sum_{e=1}^{M} V_{2}, \quad E_{\theta}=\sum_{e=1}^{N} V_{3}, \\
V_{2}=A e^{\left[\rho /\left(r_{i j}-r_{\max i j}\right)\right]}\left(B / r_{i j}^{4}-1\right), \\
V_{3}=K e^{\left[\rho_{i j} /\left(r_{i j}-r_{\max i j}\right)+\rho_{i k} /\left(r_{i k}-r_{\max i k}\right)\right]}\left(\cos \theta_{i j k}-\cos \theta_{0}\right)^{2},
\end{gathered}
$$

where $V_{2}$ corresponds to the bond-stretching and $V_{3}$ associates with the angle-bending. Potential parameters are available in [19] for P-P interaction.

DFT calculations and MD simulations are time-consuming. Molecular dynamics finite element methods (or atomic-scale finite element methods or atomistic finite element methods) have been developed to analyze nanostructured materials, see e.g. [20, 21]. Please refer to $[20,21]$ for further detailed procedure and advantages of this method. We have used MDFEM to analyze the mechanical properties of various hexagonal sheets, see e.g. [22-24]. MDFEM with Stillinger-Weber potential was implemented in our previous work [24] for phosphorene. We use here MDFEM with Stillinger-Weber potential to simulate the uniaxial tension of $\alpha$-P nanotubes. The three armchair $\alpha$-P nanotubes which have $(0,15) ;(0,19)$ and $(0,21)$ configurations with diameter from $21.706 \AA$ to $29.977 \AA$, respectively are studied. The three zigzag $\alpha$-P nanotubes which have $(20,0)$, $(26,0)$ and $(28,0)$ with diameter from $21.814 \AA$ to $29.881 \AA$, respectively are considered. The length-diameter ratios $L / D$ of all tubes are 10. The geometric parameters of $\alpha$-P are taken from [25]. $\sigma$ and $\varepsilon$ denote the nominal axial stress (engineering stress) and nominal axial strain (engineering strain), respectively. $t$ is the tube's thickness. Young's modulus $Y$ is determined from the first derivative of the stress-strain curve at $\varepsilon=0$.

\section{RESULTS AND DISCUSSION}

Fig. 2 shows the uniaxial tensile stress-strain curves of armchair and zigzag $\alpha$-P nanotubes. At a given axial strain, the axial tensile stress of the armchair phosphorene nanotube is always higher than that of the corresponding zigzag one. The axial tensile stress increases monotonously with an increase of the axial tensile strain up to a peak value, then the axial tensile stress drops suddenly for all tubes as shown in Fig. 2. This phenomenon demonstrates a brittle fracture. Hence, maximal axial stress and strain at maximal stress refer to fracture stress and fracture strain, respectively. These results are in good 
agreement with those from DFT-tight binding calculations by Sorkin et al. [10]. Our results of Young's modulus, fracture stress, fracture strain and Poisson's ratio of armchair and zigzag $\alpha$-P nanotubes are shown in Tabs. 1 and 2. Our results reveal that the fracture stress increases slightly when the tube diameter increases. In addition, the fracture strain decreases slightly from $16.5 \%$ to $16.1 \%$ when the tube diameter increases. These findings about the tendency of fracture stress of all tubes and fracture strain of armchair $\alpha$-P nanotube agree well with those from DFT-tight binding calculations by Sorkin et al. [10]. However, our study shows that the fracture strain of zigzag $\alpha$-P nanotubes increases from $18.7 \%$ to $26.0 \%$ with an increase of the tube diameter, while Sorkin et al. [10] showed an inverse tendency. This disagreement could be due to the differences in computational methods and boundary setup.

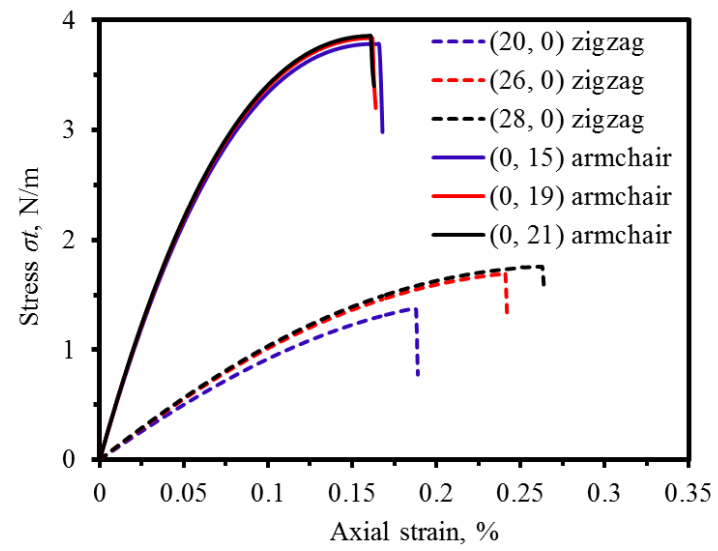

Fig. 2. The stress-strain curves of armchair and zigzag $\alpha$-P nanotubes under uniaxial tension

Table 1. The mechanical properties of armchair $\alpha$-P nanotubes by MDFEM

\begin{tabular}{ccccc}
\hline $\begin{array}{c}\text { Armchair } \\
\alpha \text {-P tube }\end{array}$ & $\begin{array}{c}\text { Young's modulus } \\
Y t, \mathrm{~N} / \mathrm{m}\end{array}$ & $\begin{array}{c}\text { Maximal stress } \\
\sigma t, \mathrm{~N} / \mathrm{m}\end{array}$ & $\begin{array}{c}\text { Strain at maximal } \\
\text { stress, } \%\end{array}$ & $\begin{array}{c}\text { Poisson's ratio } \\
(0,15)\end{array}$ \\
53.88 & 3.784 & 16.5 & 0.0324 \\
$(0,19)$ & 54.74 & 3.838 & 16.2 & 0.0336 \\
$(0,21)$ & 55.06 & 3.858 & 16.1 & 0.0436 \\
\hline
\end{tabular}

Table 2. The mechanical properties of zigzag $\alpha$-P nanotube by MDFEM

\begin{tabular}{ccccc}
\hline $\begin{array}{c}\text { Zigzag } \\
\alpha \text {-P tube }\end{array}$ & $\begin{array}{c}\text { Young's modulus } \\
Y t, \mathrm{~N} / \mathrm{m}\end{array}$ & $\begin{array}{c}\text { Maximal stress } \\
\sigma t, \mathrm{~N} / \mathrm{m}\end{array}$ & $\begin{array}{c}\text { Strain at maximal } \\
\text { stress, } \%\end{array}$ & Poisson's ratio \\
\hline$(20,0)$ & 10.65 & 1.378 & 18.7 & 0.0483 \\
$(26,0)$ & 11.72 & 1.701 & 24.0 & 0.0410 \\
$(28,0)$ & 11.96 & 1.756 & 26.0 & 0.0385 \\
\hline
\end{tabular}


In general, when the tube diameter increases, the fracture strain of $\alpha$-P nanotubes approaches that of phosphorene sheet $16 \%$ and $28 \%$ in the zigzag and armchair direction, respectively [14]) because the sheet can be considered as a tube with its infinite diameter.

Figs. 3 and 4 show the variations of the Young's modulus of armchair and zigzag $\alpha-\mathrm{P}$ nanotubes versus their diameter. The results show that Young's modulus increases monotonously with an increase of the tube diameter. This issue has been observed in MD simulations by Chen et al. [9] and in DFT-tight binding calculations by Sorkin et al. [10].

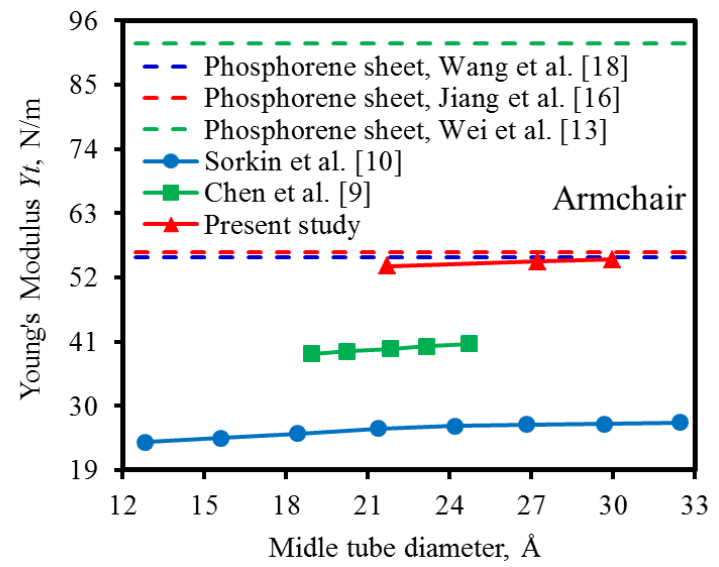

Fig. 3. The variations of the Young's modulus of armchair $\alpha$-P nanotubes versus the tube diameter

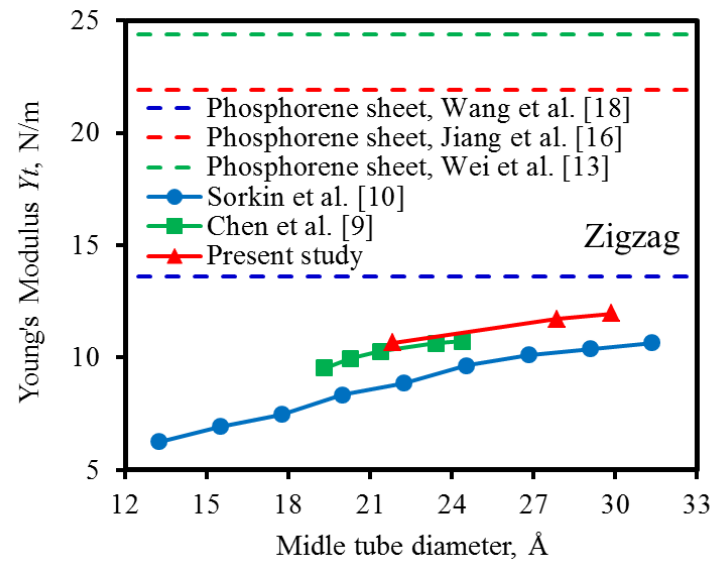

Fig. 4. The variations of the Young's modulus of zigzag $\alpha$-P nanotubes versus the tube diameter

\section{CONCLUSIONS}

The uniaxial tensile properties of 3 pairs of $\alpha$-P nanotubes are considered by MDFEM with Stillinger-Weber potential. Their Young's modulus and fracture stress increase when their diameter increases. Young's modulus of armchair phosphorene nanotube is larger than that of the corresponding zigzag one. Further studies should be conducted for a better comprehension of the mechanical properties of $\alpha$-P nanotubes. For example, the effects of missing atoms on their mechanical properties should be included in the simulation, and the range of the studied tubes (their diameters) should be larger to obtain a more general conclusion. Such issues are subjects of our future work.

\section{ACKNOWLEDGMENT}

This work was supported by Vietnam National Foundation for Science and Technology Development (NAFOSTED) under the grant number: 107.02-2017.02.

\section{REFERENCES}

[1] W. Lu, H. Nan, J. Hong, Y. Chen, C. Zhu, Z. Liang, X. Ma, Z. Ni, C. Jin, and Z. Zhang. Plasma-assisted fabrication of monolayer phosphorene and its Raman characterization. Nano Research, 7, (6), (2014), pp. 853-859. doi:10.1007/s12274-014-0446-7. 
Van-Trang Nguyen, Danh-Truong Nguyen, Minh-Quy Le

[2] L. Li, Y. Yu, G. J. Ye, Q. Ge, X. Ou, H. Wu, D. Feng, X. H. Chen, and Y. Zhang. Black phosphorus field-effect transistors. Nature Nanotechnology, 9, (5), (2014), pp. 372-377. doi:10.1038/nnano.2014.35.

[3] F. Xia, H. Wang, and Y. Jia. Rediscovering black phosphorus as an anisotropic layered material for optoelectronics and electronics. Nature Communications, 5, (2014). doi:10.1038/ncomms5458.

[4] J. Qiao, X. Kong, Z.-X. Hu, F. Yang, and W. Ji. High-mobility transport anisotropy and linear dichroism in few-layer black phosphorus. Nature Communications, 5, (2014). doi:10.1038/ncomms5475.

[5] H. Liu, A. T. Neal, Z. Zhu, Z. Luo, X. Xu, D. Tománek, and P. D. Ye. Phosphorene: an unexplored 2D semiconductor with a high hole mobility. ACS Nano, 8, (4), (2014), pp. 4033-4041. doi:10.1021/nn501226z.

[6] L. Kou, T. Frauenheim, and C. Chen. Phosphorene as a superior gas sensor: selective adsorption and distinct I-V response. The Journal of Physical Chemistry Letters, 5, (15), (2014), pp. 2675-2681. doi:10.1021/jz501188k.

[7] G. C. Guo, X. L. Wei, D. Wang, Y. Luo, and L. M. Liu. Pristine and defect-containing phosphorene as promising anode materials for rechargeable Li batteries. Journal of Materials Chemistry A, 3, (21), (2015), pp. 11246-11252. doi:10.1039/c5ta01661d.

[8] W. Li, Y. Yang, G. Zhang, and Y. W. Zhang. Ultrafast and directional diffusion of lithium in phosphorene for high-performance lithium-ion battery. Nano Letters, 15, (3), (2015), pp. 16911697. doi:10.1021/nl504336h.

[9] W. H. Chen, C. F. Yu, I. C. Chen, and H. C. Cheng. Mechanical property assessment of black phosphorene nanotube using molecular dynamics simulation. Computational Materials Science, 133, (2017), pp. 35-44. doi:10.1016/j.commatsci.2017.03.008.

[10] V. Sorkin and Y. Zhang. Mechanical properties of phosphorene nanotubes: a density functional tight-binding study. Nanotechnology, 27, (39), (2016). doi:10.1088/09574484/27/39/395701.

[11] R. Ansari, A. Shahnazari, and S. Rouhi. A density-functional-theory-based finite element model to study the mechanical properties of zigzag phosphorene nanotubes. Physica E: Low-dimensional Systems and Nanostructures, 88, (2017), pp. 272-278. doi:10.1016/j.physe.2017.01.022.

[12] I. E. Berinskii and F. M. Borodich. Elastic in-plane properties of 2D linearized models of graphene. Mechanics of Materials, 62, (2013), pp. 60-68. doi:10.1016/j.mechmat.2013.03.004.

[13] Q. Wei and X. Peng. Superior mechanical flexibility of phosphorene and few-layer black phosphorus. Applied Physics Letters, 104, (25), (2014), p. 251915. doi:10.1063/1.4885215.

[14] Z. Yang, J. Zhao, and N. Wei. Temperature-dependent mechanical properties of monolayer black phosphorus by molecular dynamics simulations. Applied Physics Letters, 107, (2), (2015). doi:10.1063/1.4926929.

[15] Z. D. Sha, Q. X. Pei, Z. Ding, J. W. Jiang, and Y. W. Zhang. Mechanical properties and fracture behavior of single-layer phosphorene at finite temperatures. Journal of Physics D: Applied Physics, 48, (39), (2015). doi:10.1088/0022-3727/48/39/395303.

[16] J. W. Jiang and H. S. Park. Mechanical properties of single-layer black phosphorus. Journal of Physics D: Applied Physics, 47, (38), (2014). doi:10.1088/0022-3727/47/38/385304.

[17] T. Hu, Y. Han, and J. Dong. Mechanical and electronic properties of monolayer and bilayer phosphorene under uniaxial and isotropic strains. Nanotechnology, 25, (45), (2014). doi:10.1088/0957-4484/25/45/455703. 
[18] C. X. Wang, C. Zhang, J. W. Jiang, H. S. Park, and T. Rabczuk. Mechanical strain effects on black phosphorus nanoresonators. Nanoscale, 8, (2), (2016), pp. 901-905. doi:10.1039/c5nr06441d.

[19] J. W. Jiang. Parametrization of Stillinger-Weber potential based on valence force field model: application to single-layer MoS2 and black phosphorus. Nanotechnology, 26, (31), (2015). doi:10.1088/0957-4484/26/31/315706.

[20] B. Liu, Y. Huang, H. Jiang, S. Qu, and K. C. Hwang. The atomic-scale finite element method. Computer Methods in Applied Mechanics and Engineering, 193, (17-20), (2004), pp. 1849-1864. doi:10.1016/j.cma.2003.12.037.

[21] J. Wackerfuß. Molecular mechanics in the context of the finite element method. International Journal for Numerical Methods in Engineering, 77, (7), (2009), pp. 969-997. doi:10.1002/nme.2442.

[22] M. Q. Le and D. T. Nguyen. The role of defects in the tensile properties of silicene. Applied Physics A, 118, (4), (2015), pp. 1437-1445. doi:10.1007/s00339-014-8904-3.

[23] M. Q. Le and D. T. Nguyen. Atomistic simulations of pristine and defective hexagonal BN and SiC sheets under uniaxial tension. Materials Science and Engineering: A, 615, (2014), pp. 481-488. doi:10.1016/j.msea.2014.07.109.

[24] D. T. Nguyen, M. Q. Le, V. T. Nguyen, and T. L. Bui. Effects of various defects on the mechanical properties of black phosphorene. Superlattices and Microstructures, 112, (2017), pp. 186199. doi:10.1016/j.spmi.2017.09.021.

[25] Y. Takao, H. Asahina, and A. Morita. Electronic structure of black phosphorus in tight binding approach. Journal of the Physical Society of Japan, 50, (10), (1981), pp. 3362-3369. doi:10.1143/jpsj.50.3362. 